\title{
Benzene containing polyhydroxyalkanoates homo- and copolymers synthesized by genome edited Pseudomonas entomophila
}

\author{
SHEN Rui ${ }^{1, \dagger}$, CAI LongWei ${ }^{1, \dagger}$, MENG DeChuan ${ }^{1}$, WU LinPing ${ }^{2}$, GUO Kai ${ }^{3}$, \\ DONG GuoXing ${ }^{4}$, LIU Lei $^{4}$, CHEN JinChun ${ }^{1}$, WU Qiong ${ }^{1} \&$ CHEN GuoQiang ${ }^{1,5^{*}}$ \\ ${ }^{1}$ MOE Key Lab of Bioinformatics, Department of Biological Science and Biotechnology, School of Life Science, Tsinghua-Peking Center for \\ Life Sciences, Tsinghua University, Beijing 100084, China; \\ ${ }^{2}$ Department of Pharmaceutics and Analytical Chemistry, Faculty of Pharmaceutical Sciences, University of Copenhagen, Copenhagen 2100, \\ Denmark; \\ ${ }^{3}$ Nanjing University of Technology, College of Biotechnology \& Pharmaceutical Engineering, Nanjing 210009, China; \\ ${ }^{4}$ Department of Chemistry, Tsinghua-Peking Center for Life Sciences, Tsinghua University, Beijing 100084, China; \\ ${ }^{5}$ Center for Nano and Micro Mechanics, Tsinghua University, Beijing 100084, China
}

Received October 17, 2013; accepted November 25, 2013; published online December 23, 2013

\begin{abstract}
Microbial synthesis of functional polymers has become increasingly important for industrial biotechnology. For the first time, it became possible to synthesize controllable composition of poly(3-hydroxyalkanoate) (P3HA) consisting of 3-hydroxydodecanoate (3HDD) and phenyl group on the side-chain when chromosome of Pseudomonas entomophila was edited to weaken its $\beta$-oxidation. Cultured in the presence of 5-phenylvaleric acid (PVA), the edited $P$. entomophila produced only homopolymer poly(3-hydroxy-5-phenylvalerate) or $\mathrm{P}(3 \mathrm{HPhV})$. While copolyesters $\mathrm{P}(3 \mathrm{HPhV}$-co-3HDD) of 3-hydroxy-5-phenylvalerate $(3 \mathrm{HPhV})$ and 3-hydroxydodecanoate (3HDD) were synthesized when the strain was grown on mixtures of PVA and dodecanoic acid (DDA). Compositions of $3 \mathrm{HPhV}$ in $\mathrm{P}(3 \mathrm{HPhV}-$ co-3HDD) were controllable ranging from $3 \%$ to $32 \%$ depending on DDDA/PVA ratios. Nuclear magnetic resonance (NMR) spectra clearly indicated that the polymers were homopolymer of $\mathrm{P}(3 \mathrm{HPhV})$ and random copolymers of $3 \mathrm{HPhV}$ and $3 \mathrm{HDD}$. Their mechanical and thermal properties varied dramatically depending on the monomer ratios. Our results demonstrated the possibility to produce tailor-made, novel functional PHA using the chromosome edited $P$. entomophila.
\end{abstract}

polyhydroxyalkanoates, PHB, Pseudomonas entomophila, $\beta$-oxidation, synthetic biology

Citation: Shen R, Cai LW, Meng DC, Wu LP, Guo K, Dong GX, Liu L, Chen JC, Wu Q, Chen GQ. Benzene containing polyhydroxyalkanoates homo- and copolymers synthesized by genome edited Pseudomonas entomophila. Sci China Life Sci, 2014, 57: 4-10, doi: 10.1007/s11427-013-4596-8

Polyhydroxyalkanoates (PHAs) are biodegradable and biocompatible polyesters synthesized by many microorganisms [1-4]. Although PHA has been developed as a type of environmentally friendly materials with low cost, it is important to introduce functionalities to PHA to improve its application [5,6]. Therefore, metabolic engineering approaches are exploited both for improving PHA production

$\uparrow$ Contributed equally to this work

*Corresponding author (email: chengq@mail.tsinghua.edu.cn) and for widening the PHA diversity [7-9]. Over 150 chiral hydroxyalkanoic acids are reported as monomers for PHA in previous investigation [5,10,11]. Generally, PHAs prepared from monomers with 3-5 (C3 to C5) carbon atoms are named as short-chain-length (SCL) PHAs, while those from monomers of $\mathrm{C} 6$ to $\mathrm{C} 14$ as medium-chain-length (MCL) PHAs [12,13]. Normally, the property of a polymer is decided by its structure [14]. Therefore, to increase the diversity of PHA structures has become a hot topic, espe- 
cially by introducing functional groups such as phenyl, halogen and unsaturated groups into PHA side chains [15-18].

Although many different organisms can utilize aromatic hydrocarbons as a carbon and energy source, only a limited number of bacteria such as Pseudomonas putida U, $P$. putida BM01 and P. oleovorans, have the ability to produce PHA-containing aromatic monomers [19-21]. P. entomophila L48, an entomopathogenic Gram-negative bacterium [22], shows a close relationship with the well-known MCL PHA producer $P$. putida. $70.2 \%$ of $P$. entomophila genes share orthologs in $P$. putida genome, of which $>96 \%$ are found in synteny. The $P$. entomophila genome harbors most of the central catabolic genes found in P. putida KT2440, indicating the possibility of MCL PHA production [23]. Furthermore, $P$. entomophila was found to contain genes encoding enzymes for the catabolism of long-chain carbohydrates. Therefore, $P$. entomophila was explored as a MCL PHA producer [24]. A $P$. entomophila strain LAC23, in which its putative chromosomal $\beta$-oxidation related genes are edited (deleted) to reduce its $\beta$-oxidation ability, is able to produce MCL PHA homopolymer of 3-hydroxydodecanoate (P3HDD) when grown in the presence of dodecanoic acid (DDA) [25-27].

In this study, we aimed to investigate the possibility of synthesizing benzene-containing PHA (homo- and random copolymers) using the genome edited $P$. entomophila strain LAC23. The benzene ring is expected to add functionality to PHA, thus allowing new possible applications.

\section{Materials and methods}

\subsection{Bacterial strains}

$P$. entomophila LAC23, a mutant of P. entomophila L48, was used in this study. Its genome was edited by removing $\beta$-oxidation related genes $\mathrm{fadBA}$ and PSEEN 0664 [22]. This mutated strain shows normal growth in the presence of DDA, and it is able to produce MCL PHA homopolymers and monomers [28-31]. Due to the weakened $\beta$-oxidation cycle, more carbon fluxes from fatty acids were directed into PHA synthesis without changing the fatty acid structures [32]. It is therefore possible that a fatty acid containing functional group(s) can be incorporated into PHA polymer chains.

\subsection{Culture media and cultivation conditions}

Seed cultures were incubated at $30^{\circ} \mathrm{C}$ in $\mathrm{LB}$ medium containing $5 \mathrm{~g} \mathrm{~L}^{-1}$ yeast extract, $10 \mathrm{~g} \mathrm{~L}^{-1}$ tryptone and $10 \mathrm{~g} \mathrm{~L}^{-1}$ $\mathrm{NaCl}$ for $12 \mathrm{~h}$ at $200 \mathrm{rmin} \mathrm{min}^{-1}$ on a rotary shaker (HNY2112B, Honor, China). They were used for a $48 \mathrm{~h}$ shake flask study carried out on the same rotary shaker at $200 \mathrm{r}$ min $^{-1}$ placed with $500 \mathrm{~mL}$ conical flasks, which contained $50 \mathrm{~mL} 4$ YLB medium supplemented with $12 \mathrm{~g} \mathrm{~L}^{-1}$ tryptone and $24 \mathrm{~g} \mathrm{~L}^{-1}$ yeast extract. Relevant fatty acids for PHA generation including DDA and PVA were added into the medium [33]. Figure 1 illustrates how the relevant PHA is produced via the engineered pathway.

To produce adequate amount of PHA for thermal and mechanical property characterization, we used $500 \mathrm{~mL}$ conical flasks containing $100 \mathrm{~mL}$ 4YLB medium supplemented with relevant fatty acids, and we also used more flasks.

\subsection{PHA extraction and purification}

Cells were harvested by centrifugation (CR21 GIII, HITACHI, Japan) at $9600 \mathrm{r} \mathrm{min}^{-1}$ for $10 \mathrm{~min}$, and were directly lyophilized. Cell dry weights (CDW) were measured after lyophilization. PHA content and PHA type were

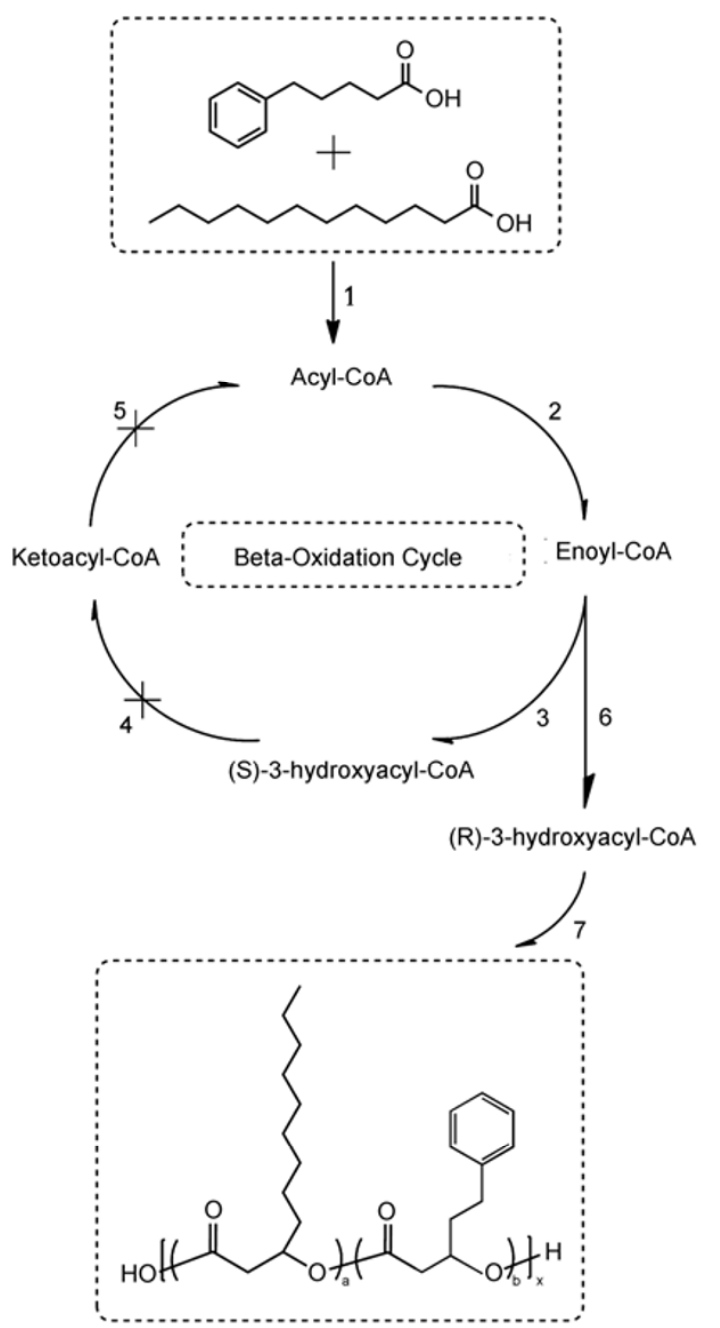

Figure 1 Major metabolic pathway for microbial synthesis of $\mathrm{P}(3 \mathrm{HPhV}-c o-3 \mathrm{HDD}) .1$ : FadD, fatty acid CoA ligase; 2: FadE, acyl-coA dehydrogenase; 3: FadBA, S-enoyl-coA hydratase; 4: FadB, 3-hydroxyacylcoA dehydrogenase; 5: FadA, 3-ketothiolase, PSEEN 0664, acetyl-coA acetyltransferase; 6: PhaJ, R-enoyl-coA hydratase; 7: PhaC, PHA synthase. 
both analyzed using gas chromatography (GC-2014, SHIMADZU, Japan) after methyl esterification in chloroform [34]. The lyophilized cells were treated with chloroform at $100^{\circ} \mathrm{C}$ for $4 \mathrm{~h}$. The intracellular PHA was obtained by Soxhlet extractor (Soxtec 2050, Foss, Denmark), and PHA was dissolved in chloroform and precipitated in an excess of 10 volumes of ethanol. The solution containing PHA precipitates was centrifuged at $8000 \mathrm{r} \mathrm{min}^{-1}$ for 10 min. After the supernatant was discarded, the purified PHA was dissolved in chloroform for film casting, and all solvents were evaporated for $7 \mathrm{~d}$ at room temperature to consolidate the crystallization [26].

\subsection{NMR analysis of PHA}

The ${ }^{1} \mathrm{H}$ and ${ }^{13} \mathrm{C}$ spectra were performed with a JEOL JNMECA 600 NMR spectrometer to determine the polymer composition, the chemical microstructure and the monomer sequences. Tetramethylsilane was used as the internal standard [35].

\subsection{Characterization of PHA physical properties}

Molecular weights were studied using gel permeation chromatography equipped with a refractive index detector (RID-10A, SHIMADZU, Japan). The measurements were carried out at $40^{\circ} \mathrm{C}$ with a SHIMADZU GPC-804C column. Differential scanning calorimetry data were recorded in the temperature range of $-80^{\circ} \mathrm{C}$ to $200^{\circ} \mathrm{C}$ under a nitrogen flow rate of $50 \mathrm{~mL} \mathrm{~min}{ }^{-1}$ on a TA instruments (DSC-Q20, TA, USA) [12]. PHA samples were casted into films by the conventional solvent-casting method for mechanical properties studies [36]. Subsequently, the PHA films were cut into dumbbell-shaped specimens with a width of $4 \mathrm{~mm}$ and a thickness of approximately $100 \mathrm{~mm}$ [36]. The stress-strain measurements of films were carried out using servo control system universal testing machine (AI-7000s, GOTECH, Taiwan, China) at room temperature.

\section{Results}

\subsection{Production of P3HPhV homopolymer by $P$. ento- mophila LAC23}

P. entomophila LAC23 was able to produce P(3-hydroxy5-phenylvalerate) or $\mathrm{P} 3 \mathrm{HPhV}$ homopolymer when PVA was added into culture medium. $\mathrm{P} 3 \mathrm{HPhV}$ was accumulated to around $4.86 \mathrm{wt} \%$ of the cell dry weight (Table 1). However, the $\mathrm{P} 3 \mathrm{HPhV}$ content was very low. $\mathrm{P} 3 \mathrm{HPhV}$ turned out to be a highly amorphous and sticky material after extraction, which limits its application. Therefore, it was necessary to enhance its rigid properties via copolymerization with another monomer. Since P(3-hydroxydodecanoate) or P3HDD produced by $P$. entomophila has strong mechanical properties [25], 3-hydroxydodecanoate (3HDD) was chosen as a copolymer monomer with 3-hydroxy-5-phenylvalerate $(3 \mathrm{HPhV})$ to make a mechanically useful material.

\subsection{Production of $\mathrm{P}(3 \mathrm{HPhV}-\mathrm{co}-3 \mathrm{HDD})$ by $P$. ento- mophila LAC23}

A mixture of PVA and DDA in cultures of the P. entomophila LAC23 led to the formation of copolyester $\mathrm{P}(3 \mathrm{HPhV}-\mathrm{co}-3 \mathrm{HDD})$ consisting of $3 \mathrm{HPhV}$ and $3 \mathrm{HDD}$. Composition of the monomers can be adjusted by changing the ratio of DDA to PVA. For example, $17 \mathrm{wt} \% \mathrm{P}(21 \mathrm{~mol} \%$ $3 \mathrm{HPhV}-\mathrm{co}-79 \mathrm{~mol} \%$ 3HDD) was accumulated when DDA/PVA ratio was $2 / 3$, while a ratio of $0.5 / 3$ (or $1 / 6$ ) resulted in the formation of $15 \mathrm{wt} \% \mathrm{P}(63 \mathrm{~mol} \% 3 \mathrm{HPhV}-\mathrm{co}$ $37 \mathrm{~mol} \%$ 3HDD) (Table 1). Interestingly, the transparency of $\mathrm{P}(3 \mathrm{HPhV}$-co-3HDD) was dependent on monomer compositions, as increasing $3 \mathrm{HPhV}$ percentage in the copolymers softened the copolymer and reduced its transparency (Figure 2). The highest transparency was found to be with $\mathrm{P}(3 \mathrm{HPhV}-$ co-97mol\% 3HDD).

\subsection{NMR microstructure analysis}

The chemical structure of this novel PHA homopolymer

Table $1 \mathrm{P}\left(3 \mathrm{HPhV}\right.$-co-3HDD) production by $P$. entomophila LAC23 grown in shake flasks containing PVA and/or DDA ${ }^{\text {a) }}$

\begin{tabular}{|c|c|c|c|c|c|c|}
\hline $\operatorname{PVA}\left(\mathrm{g} \mathrm{L}^{-1}\right)$ & $\operatorname{DDA}\left(\mathrm{g} \mathrm{L}^{-1}\right)$ & $\mathrm{CDW}\left(\mathrm{g} \mathrm{L}^{-1}\right)$ & PHA $\left(\mathrm{g} \mathrm{L}^{-1}\right)$ & PHA/CDW (wt $\%$ ) & $3 \mathrm{HPhV}(\mathrm{mol} \%)$ & $3 \mathrm{HDD}(\mathrm{mol} \%)$ \\
\hline 3 & 0 & $4.34 \pm 0.31$ & $0.21 \pm 0.06$ & $4.86 \pm 1.13$ & 100 & 0 \\
\hline 3 & 0.5 & $4.84 \pm 0.40$ & $0.65 \pm 0.05$ & $13.44 \pm 0.20$ & $63.37 \pm 0.96$ & $36.63 \pm 0.96$ \\
\hline 3 & 1 & $5.26 \pm 0.45$ & $0.97 \pm 0.07$ & $18.55 \pm 0.49$ & $39.84 \pm 0.72$ & $60.16 \pm 0.72$ \\
\hline 3 & 2 & $5.94 \pm 0.68$ & $1.10 \pm 0.02$ & $17.09 \pm 0.14$ & $21.49 \pm 0.60$ & $78.51 \pm 0.60$ \\
\hline
\end{tabular}

a) P. entomophila LAC23 was cultivated in 4YLB medium supplemented with different concentrations of PVA and/or DDA for 48 h. Data shown are the averages and standard deviations of three parallel experiments. Abbreviations: PVA, 5-phenylvaleric acid; DDA, dodecanoic acid; CDW, cell dry weight. 3HPhV, 3-hydroxy-5-phenylvalerate; 3HDD, 3-hydroxydodecanoate. 

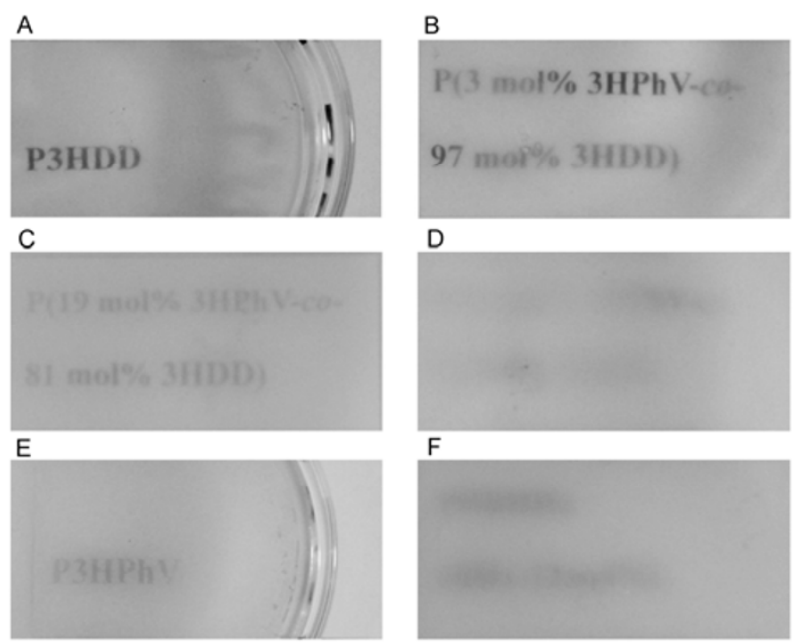

Figue 2 Transparencies of $\mathrm{P}(3 \mathrm{HPhV}-\mathrm{co}-3 \mathrm{HDD})$ films consisting of different monomer compositions. The films were placed $1.5 \mathrm{~cm}$ above white papers printed with polymer names. A, P3HDD. B, P(3HPhV-co-97mol\% 3HDD). C, P(3HPhV-co-81mol\% 3HDD). D, P(3HPhV-co-68mol\% 3HDD). E, P3HPhV. F, PHBHHx (3HHX: $12 \mathrm{~mol} \%$, control group) of 3-hydroxybutyrate and 3-hydroxyhexanoate (3HHx).

containing aromatic side chain is shown in Figure 3. The structure of homopolymer poly(3-hydroxy-5-phenylvalerate) $(\mathrm{P}(3 \mathrm{HPhV}))$ was confirmed by NMR studies (Figure 4). From the ${ }^{1} \mathrm{H}$ NMR and ${ }^{13} \mathrm{C}$ NMR spectra, $\mathrm{P}(3 \mathrm{HPhV})$ has the following characteristics: ${ }^{1} \mathrm{H} \mathrm{NMR}\left(\mathrm{CDCl}_{3}\right): \delta=1.94(\mathrm{~m} ; 2 \mathrm{H}$, H-4), 2.52-2.59 (m; 2H, H-2), 2.62 (m; 2H, H-5), 5.28 (m; 1H, H-3), 7.18 (m; 3H, H-7 and H-9), 7.28 (m; 2H, H-8) (Figure $4 \mathrm{~A}) ;{ }^{13} \mathrm{C} \mathrm{NMR}\left(\mathrm{CDCl}_{3}\right): \delta=31.56(\mathrm{C}-5), 35.52(\mathrm{C}-4)$, 39.15 (C-2), 70.63 (C-3), 126.22 (C-9), 128.47 (C-7), 128.63 (C-8), 141.09 (C-6), 169.43 (C-1) (Figure 4B). This result was similar to previous study [37], allowing us to conclude that the monomer of this PHA was indeed HPhV.

The microstructures of copolyester $\mathrm{P}(3 \mathrm{HPhV}-\mathrm{co}-3 \mathrm{HDD})$ were also studied using NMR (Figures 4 and 5). ${ }^{1} \mathrm{H}$ NMR and ${ }^{13} \mathrm{C}$ NMR spectra of $\mathrm{P}(3 \mathrm{HPhV}-$ co-3HDD) containing $32 \mathrm{~mol} \% 3 \mathrm{HPhV}$ were collected. Based on the chemical shift assignment for each proton (Figure 6A) and for carbon resonance (Figure 6B), the polymer was confirmed to be a random copolymer. Together with the well-characterized proton resonances in 3HDD units, two proton resonance peaks $(\mathrm{PhV}(7,8), \mathrm{PhV}(9))$ appeared with identical intensities which are assigned to the hydrogen on the benzene ring of $3 \mathrm{HPhV}$ units [37]. The expanded spectra of individual splitting resonance of each carbon in random copolymer $\mathrm{P}(3 \mathrm{HDD}-\mathrm{co}-3 \mathrm{HPhV}$ ) were clearly revealed (Figure 6B). The detailed assignment was referred to previous studies on mcl-PHA [38] and biodegradable aromatic plastics from a bacterial source [37]. All carbon resonances were split into several peaks because of the strong interaction of 3HDD and $3 \mathrm{HPhV}$ units in the copolymer. This phenomenon can boil down to center on triad co-monomer sequences of two

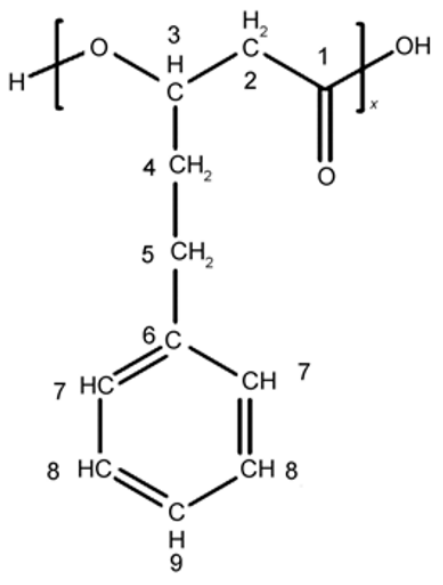

Figure 3 Chemical structure of poly(3-hydroxy-5-phenylvaleric acid) or $\mathrm{P}(3 \mathrm{HPhV})$.

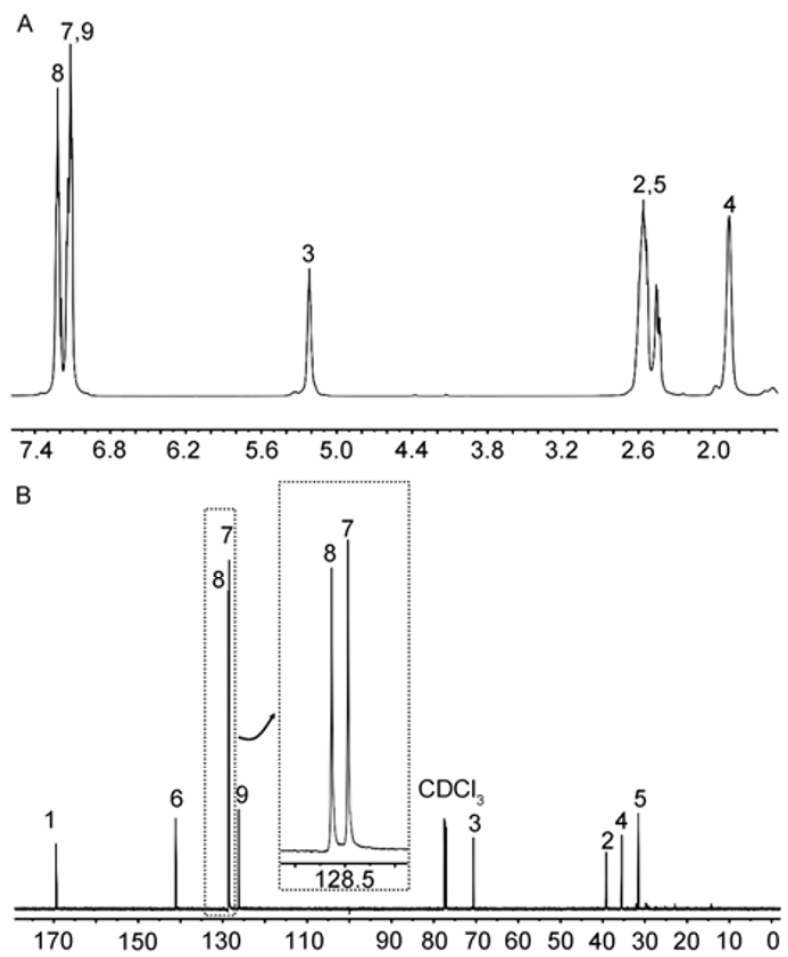

Figure $4{ }^{1} \mathrm{H}$ NMR (A) and ${ }^{13} \mathrm{C}$ NMR (B) spectra of homopolymer poly(3-hydroxy-5-phenylvaleric acid). Chemical shifts are in ppm and tetramethylsilane (TMS) is used as an internal chemical shift standard. Numbering scheme refers to Figure 3.

monomers $3 \mathrm{HDD}$ and $3 \mathrm{HPhV}$, which is quite common in random PHA copolymer [3,12,39]. These analyses provided solid evidence that this PHA polymer is a random $\mathrm{P}(3 \mathrm{HDD}-$ co-3HPhV) copolymer.

\subsection{Physical characterization of $\mathrm{P}(3 \mathrm{HPhV}-$ co-3HDD $)$ copolymers}

A series of $\mathrm{P}(3 \mathrm{HPhV}-c o-3 \mathrm{HDD})$ synthesized by $P$. ento- 


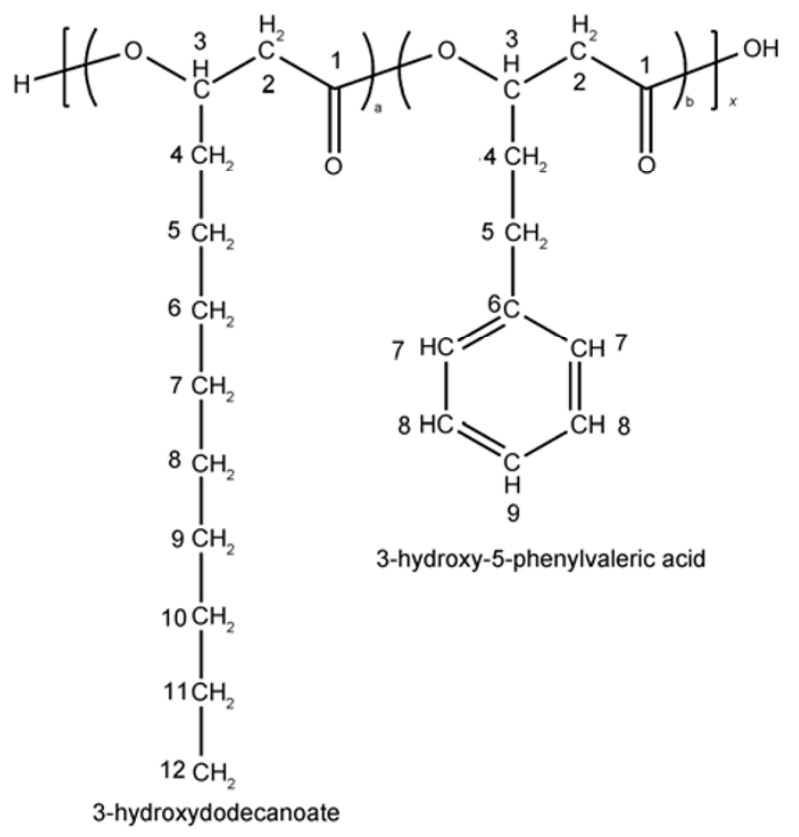

Figure 5 Chemical structure of poly(3-hydroxydodecanoate-co-3- hydroxy-5-phenylvaleric acid) or P(3HDD-co-3HPhV).

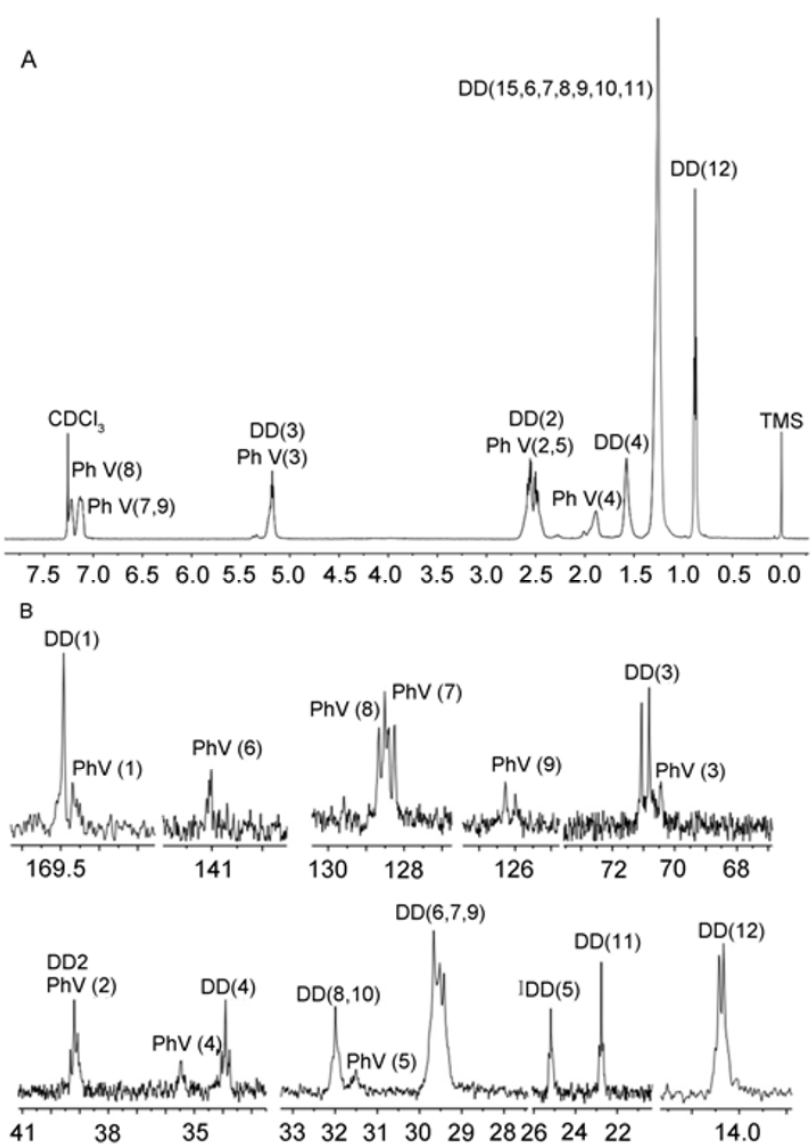

Figure $6{ }^{1} \mathrm{H}$ NMR (A) and ${ }^{13} \mathrm{C}$ NMR (B) spectra of random copolymer $\mathrm{P}(3 \mathrm{HDD}-\mathrm{co}-3 \mathrm{HPhV})$. DD and $\mathrm{PhV}$ refer to $3 \mathrm{HDD}$ and $3 \mathrm{HPhV}$. The molar ratio of $3 \mathrm{HDD}$ and $3 \mathrm{HPhV}$ is $68.03 \%$ and $31.97 \%$, respectively. Chemical shifts are in ppm and tetramethylsilane (TMS) is used as an internal chemical shift standard. Numbering scheme refers to Figure 5. mophila LAC23 as described above (Table 1) were extracted, purified, and cut into dumbbell-shaped specimens for the following thermal and mechanical property studies. At the same time, gel permeation chromatography study showed that homopolymer $\mathrm{P} 3 \mathrm{HPhV}$ has the lowest weight-average molecular weights $\left(M_{w}\right)$ and number average-molecular weights $\left(M_{n}\right)$ among all $\mathrm{P}(3 \mathrm{HPhV}-$ co-3HDD) and homopolymer P3HDD. Additionally, $\mathrm{P} 3 \mathrm{HPhV}$ had the widest molecular weight distribution $M_{w} / M_{n}$, and P3HDD homopolymer had the highest $M_{w}$ and $M_{n}$ (Table 2).

The addition of $3 \mathrm{HDD}$ into $\mathrm{P} 3 \mathrm{HPhV}$ resulted in an apparent decrease on the glass transition temperature $\left(T_{g}\right)$ from $6^{\circ} \mathrm{C}$ to around $-35^{\circ} \mathrm{C}$ and clearly increased the melting temperature $\left(T_{m}\right)$ from $50^{\circ} \mathrm{C}$ to around $80^{\circ} \mathrm{C}$ when $3 \mathrm{HDD}$ ratio rose from $0 \mathrm{~mol} \%$ to $68-97 \mathrm{~mol} \%$ (Table 3 ). The $T_{m}$ and $T_{g}$ values were estimated by the result of the first and second heating scan, respectively. Therefore, the thermal property of copolyester containing phenyl group can be easily modified by changing the monomer composition, which leads to a moderate property between the two homopolymers.

$\mathrm{P} 3 \mathrm{HPhV}$ homopolymer is a sticky material even at room temperature. Its random copolymerization with 3HDD resulted in a lower yield strength $\left(\sigma_{y}\right)$, maximum tension strength $\left(\sigma_{t}\right)$ and elongation at break $\left(\varepsilon_{b}\right)$ than P3HDD homopolymer (Table 3). Interestingly, $\mathrm{P}(3 \mathrm{HPhV}$-co-3HDD) with $18.70 \mathrm{~mol} \% 3 \mathrm{HPhV}$ presented a higher $\varepsilon_{b}$ than P3HDD, indicating a non-linear relationship between $3 \mathrm{HPhV}$ content and properties. On the other hand, the Young's modulus $(E)$ of the copolyester became higher than that of P3HDD, except $\mathrm{P}(3 \mathrm{HPhV}$-co-3HDD) with $31.97 \mathrm{~mol} \% 3 \mathrm{HPhV}$.

\section{Discussion}

Phenyl group was introduced for the first time into the PHA homopolymer P3HDD by genome edited P. entomophila, bringing changes to the thermal and mechanical properties of P3HDD. This strain is a mutant of P. entomophila L48, in which the key $\beta$-oxidation genes $\mathrm{fadA}$, fadB and PSEEN 0664 were deleted (Figure 1). Deletion of these acetyltranferase encoding genes results in weakened $\beta$-oxidation,

Table 2 Molecular weights of $\mathrm{P} 3 \mathrm{HPhV}, \mathrm{P} 3 \mathrm{HDD}$ and $\mathrm{P}(3 \mathrm{HPhV}-$ co$3 \mathrm{HDD})^{\mathrm{a})}$

\begin{tabular}{|c|c|c|c|}
\hline $\begin{array}{c}\mathrm{P}(3 \mathrm{HDD}-\mathrm{co}-3 \mathrm{HPhV}) \\
3 \mathrm{HPhV}(\mathrm{mol} \%)\end{array}$ & $M_{n}\left(10^{4} \mathrm{Da}\right)$ & $M_{w}\left(10^{4} \mathrm{Da}\right)$ & $M_{w} / M_{n}$ \\
\hline 0 & 5.2 & 10.4 & 2.0 \\
\hline 2.91 & 4.1 & 6.56 & 1.6 \\
\hline 18.70 & 4.3 & 7.31 & 1.7 \\
\hline 31.97 & 3.4 & 6.12 & 1.8 \\
\hline 100 & 2.1 & 4.41 & 2.1 \\
\hline
\end{tabular}

a) Molecular weights were studied using gel permeation chromatography. Abbreviations: $M_{w}$, weight-average molecular weight; $M_{n}$, number-average molecular weight. 
Table 3 Physical characterization of the microbial $\mathrm{P}(3 \mathrm{HPhV}-\mathrm{co}-3 \mathrm{HDD})^{\mathrm{a})}$

\begin{tabular}{|c|c|c|c|c|c|c|}
\hline \multirow{2}{*}{$\begin{array}{c}\mathrm{P}(3 \mathrm{HDD}-c o-3 \mathrm{HPhV}) 3 \mathrm{HPhV} \\
(\mathrm{mol} \%)\end{array}$} & \multicolumn{2}{|c|}{ Thermal properties } & \multicolumn{4}{|c|}{ Mechanical properties } \\
\hline & $T_{m}\left({ }^{\circ} \mathrm{C}\right)$ & $T_{g}\left({ }^{\circ} \mathrm{C}\right)$ & $\sigma_{y}(\mathrm{MPa})$ & $\sigma_{t}(\mathrm{MPa})$ & $\varepsilon_{b}(\%)$ & $E(\mathrm{MPa})$ \\
\hline 0 & 82.4 & -49.3 & $5.5 \pm 0.8$ & $5.5 \pm 0.9$ & $60 \pm 34$ & $61.1 \pm 6.4$ \\
\hline 2.91 & 81.00 & -33.35 & $1.53 \pm 0.65$ & $2.05 \pm 0.51$ & $37.38 \pm 6.28$ & $93.91 \pm 20.52$ \\
\hline 18.70 & 80.13 & -35.81 & $3.63 \pm 0.68$ & $4.36 \pm 0.94$ & $86.03 \pm 39.80$ & $94.79 \pm 34.95$ \\
\hline 31.97 & 75.84 & -35.15 & $2.84 \pm 1.05$ & $3.15 \pm 1.21$ & $32.02 \pm 15.94$ & $48.72 \pm 24.04$ \\
\hline 100 & 50.40 & 5.90 & - & - & - & - \\
\hline
\end{tabular}

a) $T_{m}$, melting temperature; $T_{g}$, glass transition temperature; $\sigma_{y}$, yield strength; $\sigma_{t}$, maximum tension strength; $\varepsilon_{b}$, elongation at break; $E$, Young's modulus.

thus allowing more carbon fluxes being directed into PHA synthesis [25].

The successful synthesis of $\mathrm{P}(3 \mathrm{HPhV}-$ co-3HDD) indicates that genome edited $P$. entomophila is able to add functionalities to PHA. Functionalities provided by the phenyl group in the copolymer are expected to add value to PHA, as shown in this study, transparency of PHA was decreased by the increasing presence of $3 \mathrm{HPhV}$ in the copolymers. More novel properties are to be expected from this new material.

PHA commercialization began from early 1980s, although it has been a slow and painful process. PHA with their biodegradability and sustainability has suffered from high production cost, making it difficult to compete with petrochemical based plastics [1]. Various applications including chiral drug intermediates, biomedicines and tissue engineering, have been exploited [5], yet bureaucratic approval processes for medical usages slowed down the development. Biofuel application of PHA will also be restricted by its high cost of handling [40].

Therefore, it becomes increasingly important to add values to the expensive PHA. We think that high-value-added properties including ultra strength, shape memory, gas or liquid permeable selectivity, self healing, light wave length absorption selectivity and controllable degradation, are desirable properties for high-value-added applications. To synthesize materials with these properties, people have to introduce functional groups into the materials without being damaged during the synthesis processes.

Since most of the chemical processes including the polymerization are conducted at a high temperature, functional groups have to be protected and de-protected, which requires complete organic processing and harsh conditions. In contrast, microbial processes are carried out under gentle aqueous conditions, and functional groups survive more easily without protection. Thus, microbial processing will be a favorable approach for introducing active groups into a polymer chain, as also evidenced in this study.

However, due to the $\beta$-oxidation process of microorganisms, functional groups containing fatty acids as precursors for PHA synthesis will be mostly removed or destructed, leading to failure on functional groups incorporation into the polymer chains.

Now that $\beta$-oxidation ability in $P . \mathrm{sp}$ is weakened
[25,29,30,36], fatty acids with or without functional groups can be polymerized in their original structures to become a PHA with structures precisely the same as the fatty acids we added, we are entering an era of functional PHA production with high-value-added properties.

A new functional PHA research high tide is about to come.

This work was supported by the National High Technology Research and Development Program of China (2012AA023102 to Liu Lei, Guo Kai and Wu Qiong), the National Basic Research Program of China (2012CB725201 to Chen GuoQiang and Chen JinChun, 2012CB725204 to Guo Kai and Wu Qiong), and National Natural Science Foundation of China (31270146 to Chen GuoQiang).

1 Chen GQ, Patel MK. Plastics derived from biological sources: Present and future: A technical and environmental review. Chem Rev, 2012, 112: 2082-2099

2 Kim DY, Kim HW, Chung MG, Rhee YH. Biosynthesis, modification, and biodegradation of bacterial medium-chain-length polyhydroxyalkanoates. J Microbiol, 2007, 45: 87-97

3 Anderson AJ, Dawes EA. Occurrence, metabolism, metabolic role, and industrial uses of bacterial polyhydroxyalkanoates. Microbiol Rev, 1990, 54: 450-472

4 Steinbüchel A, Fuchtenbusch B. Bacterial and other biological systems for polyester production. Trends Biotechnol, 1998, 16: 419-427

5 Chen GQ. A microbial polyhydroxyalkanoates (PHA) based bio- and materials industry. Chem Soc Rev, 2009, 38: 2434-2446

6 Gao X, Chen JC, Wu Q, Chen GQ. Polyhydroxyalkanoates as a source of chemicals, polymers, and biofuels. Curr Opin Biotechnol, 2011, 22: 768-774

7 Carole TM, Pellegrino J, Paster MD. Opportunities in the industrial biobased products industry. Appl Biochem Biotechnol, 2004, 113: $871-885$

8 Hazer B, Steinbüchel A. Increased diversification of polyhydroxyalkanoates by modification reactions for industrial and medical applications. Appl Microbiol Biotechnol, 2007, 74: 1-12

9 Keshavarz T, Roy I. Polyhydroxyalkanoates: Bioplastics with a green agenda. Curr Opin Microbiol, 2010, 13: 321-326

10 Hu D, Chung AL, Wu LP, Zhang X, Wu Q, Chen JC, Chen GQ. Biosynthesis and characterization of polyhydroxyalkanoate block copolymer P3HB- $b$-P4HB. Biomacromolecules, 2011, 12: 3166-3173

11 Verlinden RAJ, Hill DJ, Kenward MA, Williams CD, Radecka I. Bacterial synthesis of biodegradable polyhydroxyalkanoates. J Appl Microbiol, 2007, 102: 1437-1449

12 Meng DC, Shi ZY, Wu LP, Zhou Q, Wu Q, Chen JC, Chen GQ. Production and characterization of poly(3-hydroxypropionate-co-4hydroxybutyrate) with fully controllable structures by recombinant Escherichia coli containing an engineered pathway. Metab Eng, 2012, 
14: $317-324$

13 Sudesh K, Doi Y. Molecular design and biosynthesis of biodegradable polyesters. Polym Adv Technol, 2000, 11: 865-872

14 Chanprateep S. Current trends in biodegradable polyhydroxyalkanoates. J Biosci Bioeng, 2010, 110: 621-632

15 Tobin KM, O'Connor KE. Polyhydroxyalkanoate accumulating diversity of Pseudomonas species utilising aromatic hydrocarbons. FEMS Microbiol Lett, 2005, 253: 111-118

16 Kim DY, Kim YB, Rhee YH. Evaluation of various carbon substrates for the biosynthesis of polyhydroxyalkanoates bearing functional groups by Pseudomonas putida. Int J of Biol Macromol, 2000, 28: 23-29

17 Rahayu A, Zaleha Z, Yahya ARM, Majid MIA, Amirul AA. Production of copolymer poly(3-hydroxybutyrate-co-4-hydroxybutyrate) through a one-step cultivation process. World J Microbiol Biotechnol, 2008, 24: 2403-2409

18 Olivera ER, Arcos M, Naharro G, Luengo JM. Unusual PHA biosynthesis. In: CHEN G-Q. Plastics from bacteria. Springer Berlin Heidelberg. 2010. 133-186

19 Abraham GA, Gallardo A, San Roman J, Olivera ER, Jodra R, García B, Miñambres B, García JL, Luengo JM. Microbial synthesis of poly( $\beta$-hydroxyalkanoates) bearing phenyl groups from Pseudomonas putida: Chemical structure and characterization. Biomacromolecules, 2001, 2: 562-567

20 Ishida K, Wang Y, Inoue Y. Comonomer unit composition and thermal properties of poly(3-hydroxybutyrate-co-4-hydroxybutyrate)s biosynthesized by Ralstonia eutropha. Biomacromolecules, 2001, 2: 1285-1293

21 Koller M, Bona R, Chiellini E, Fernandes EG, Horvat P, Kutschera C, Hesse P, Braunegg G. Polyhydroxyalkanoate production from whey by Pseudomonas hydrogenovora. Bioresour Technol, 2008, 99: 4854-4863

22 Vodovar N, Vallenet D, Cruveiller S, Rouy Z, Barbe V, Acosta C, Cattolico L, Jubin C, Lajus A, Segurens B, Vacherie B, Wincker P, Weissenbach J, Lemaitre B, Médigue C, Boccard F. Complete genome sequence of the entomopathogenic and metabolically versatile soil bacterium Pseudomonas entomophila. Nat Biotechnol, 2006, 24: 673-679

23 Velazquez F, Pfluger K, Cases I, De Eugenio LI, De Lorenzo V. The phosphotransferase system formed by PtsP, PtsO, and PtsN proteins controls production of polyhydroxyalkanoates in Pseudomonas putida. J Bacteriol, 2007, 189: 4529-4533

24 Sun Z, Ramsay JA, Guay M, Ramsay BA. Fermentation process development for the production of medium-chain-length poly-3hyroxyalkanoates. Appl Microbiol Biotechnol, 2007, 75: 475-485

25 Chung AL, Jin HL, Huang LJ, Ye HM, CHen JC, Wu Q, Chen GQ. Biosynthesis and characterization of poly(3-hydroxydodecanoate) by $\beta$-oxidation inhibited mutant of Pseudomonas entomophila L48. Biomacromolecules, 2011, 12: 3559-3566

26 Ouyang SP, Luo RC, Chen SS, Liu Q, Chung AL, Wu Q, Chen GQ. Production of polyhydroxyalkanoates with high 3-hydroxydodecanoate monomer content by $f a d B$ and $f a d A$ knockout mutant of Pseudomonas putida KT2442. Biomacromolecules, 2007, 8: 2504-2511
27 Sun Z, Ramsay JA, Guay M, Ramsay BA. Carbon-limited fed-batch production of medium-chain-length polyhydroxyalkanoates from nonanoic acid by Pseudomonas putida KT2440. Appl Microbiol Biotechnol, 2007, 74: 69-77

28 Zinn M. Biosynthesis of medium-chain-length poly[(r)-3hydroxyalkanoates]. In: CHEN G-Q. Plastics from bacteria. Springer Berlin Heidelberg. 2010. 213-236

29 Chung A, Liu Q, Ouyang SP, Wu Q, Chen GQ. Microbial production of 3-hydroxydodecanoic acid by PHA operon and fadBA knockout mutant of Pseudomonas putida KT2442 harboring tesb gene. Appl Microbiol Biotechnol, 2009, 83: 513-519

30 Ma L, Zhang HF, Liu Q, Chen J, Zhang J, Chen GQ. Production of two monomer structures containing medium-chain-length polyhydroxyalkanoates by $\beta$-oxidation-impaired mutant of Pseudomonas putida KT2442. Bioresour Technol, 2009, 100: 4891-4894

31 Snell KD, Feng F, Zhong LH, Martin D, Madison LL. YfcX enables medium-chain-length poly(3-hydroxyalkanoate) formation from fatty acids in recombinant Escherichia coli fadB strains. J Bacteriol, 2002, 184: 5696-5705

32 Ren Q, De Roo G, Ruth K, Witholt B, Zinn M, Thöny-Meyer L. Simultaneous accumulation and degradation of polyhydroxyalkanoates: Futile cycle or clever regulation? Biomacromolecules, 2009, 10: 916-922

33 Zhou Q, Shi ZY, Meng DC, Wu Q, Chen JC, Chen GQ. Production of 3-hydroxypropionate homopolymer and poly(3-hydroxy- propionateco-4-hydroxybutyrate) copolymer by recombinant Escherichia coli. Metab Eng, 2011, 13: 777-785

34 Kato M, Bao HJ, Kang CK, Fukui T, Doi Y. Production of a novel copolyester of 3-hydroxybutyric acid and medium chain length 3-hydroxyalkanaic acids by Pseudomonas sp 61-3 from sugars. Appl Microbiol Biotechnol, 1996, 45: 363-370

35 Tripathi L, Wu LP, Meng D, Chen JC, Chen GQ. Biosynthesis and characterization of diblock copolymer of $\mathrm{p}$ (3-hydroxypropionate)block-p(4-hydroxybutyrate) from recombinant Escherichia coli. Biomacromolecules, 2013, 14: 862-870

36 Li SY, Dong CL, Wang SY, Ye HM, Chen GQ. Microbial production of polyhydroxyalkanoate block copolymer by recombinant Pseudomonas putida. Appl Microbiol Biotechnol, 2011, 90: 659-669

37 Garcia B, Olivera ER, Minambres B, Fernández-Valverde M, Cañedo M, Prieto MA, García JL, Martínez M, Luengo JM. Novel biodegradable aromatic plastics from a bacterial source. Genetic and biochemical studies on a route of the phenylacetyl-CoA catabolon. J Biol Chem, 1999, 274: 29228-29241

38 Matsusaki H, Abe H, Doi Y. Biosynthesis and properties of poly(3-hydroxybutyrate-co-3-hydroxyalkanoates) by recombinant strains of Pseudomonas sp. 61-3. Biomacromolecules, 2000, 1: $17-22$

39 Abe H, Doi Y, Fukushima T, Eya H. Biosynthesis from gluconate of a random copolyester consisting of 3-hydroxybutyrate and medium-chain-length 3-hydroxyalkanoates by Pseudomonas sp. 61-3. Int J Biol Macromol, 1994, 16: 115-119

4040 Zhang XJ, Luo RC, Wang Z, Deng Y, CHen GQ. Applications of (R)-3-hydroxyalkanoate methyl esters derived from microbial polyhydroxyalkanoates as novel biofuel. Biomacromolecules, 2009, 10: 707-711

Open Access This article is distributed under the terms of the Creative Commons Attribution License which permits any use, distribution, and reproduction in any medium, provided the original author(s) and source are credited. 